\title{
Piratería nabatea en el Mar Rojo
}

\author{
Nabataean Piracy in the Red Sea
}

\author{
Carmen Blánquez Pérez*
}

\begin{abstract}
RESUMEN
Las actividades marítimas de los nabateos han sido poco estudiadas, a pesar de que aparecen mencionadas en las fuentes antiguas. En el presente artículo se revisan las distintas hipótesis planteadas por los historiadores actuales, que se centran en el declive del comercio nabateo y en el descubrimiento de los monzones. Sin embargo, no existió un declive comercial que justificara las actividades de piratería de los nabateos, y el descubrimiento de los monzones no se corresponde con el período en que dichas actividades de piratería tuvieron lugar.
\end{abstract}

\section{PALABRAS CLAVE:}

Comercio Nabateo. Pirateria nabatea. Descubrimiento de los Monzones.

\begin{abstract}
Maritime activities of the Nabataeans are poorly understood, although are mentioned in ancient sources. In this article we review the various hypotheses raised by modern historians, who focus on the Nabatean trade decline and the discovery of the monsoons. However, there was no declining sales to justify piracy activities of the Nabataeans, and the discovery of the monsoon does not correspond to the period in which these pirate activities took place.
\end{abstract}

\section{KEYWORDS:}

Nabataean Trade. Nabataean Piracy. Discovery of the Monsoons.

Los nabateos son conocidos principalmente por sus actividades comerciales, pues Petra fue una de las más importantes ciudades caravaneras del Mundo Antiguo, gracias a su emplazamiento en un lugar estratégico al que llegaban las rutas que provenían del Oriente y sur de Arabia y desde donde partían hacia el norte, Mediterráneo y Egipto. Su papel como intermediaria, especialmente en el comercio de los productos aromáticos (incienso y mirra) fue una de las bases esenciales de su economía y riqueza.

\footnotetext{
* Universidad Complutense de Madrid. E-mail: cblanquez@ghis.ucm.es
} 
Todo esto aparece documentado por las fuentes antiguas y sigue siendo destacado por los investigadores actuales.

Sin embargo, existe una cita de Diodoro de Sicilia (una de nuestras principales fuentes de información para el conocimiento de los nabateos, quien sigue en esta ocasión probablemente a Agatárquides de Cnido) que resulta un tanto desconcertante y sobre la que no se ha investigado debidamente:

«Una vez que se navega pasado este país se llega al Golfo Laeanites, alrededor del cual hay muchas aldeas habitadas por árabes que son conocidos como nabateos. Esta tribu ocupa una gran parte de la costa y no poco de la región que se extiende tierra adentro y tiene numerosas gentes incontables y manadas y rebaños en multitudes increíbles. Ahora bien en tiempos antiguos estos hombres respetaban la justicia y se contentaban con la comida que recibían de sus rebaños pero más tarde, después de que los reyes en Alejandría hicieron los caminos del mar navegables para sus comerciantes, estos árabes no sólo asaltaban a los náufragos, sino que equipados con barcos piratas atacaban a los viajeros, imitando en sus prácticas las maneras salvajes y sin ley del Tauro del Ponto (Mar Negro); no obstante, a veces, después eran capturados en el mar por algunas cuadrirremes y castigados como merecían» (III, 43,4-5).

Asimismo, Estrabón, otra de las fuentes relevantes para nuestro conocimiento de los nabateos, parece hacer referencia al mismo tema cuando afirma:

«Estos nabateos anteriormente vivían una vida pacífica, pero después por medio de balsas saqueaban los barcos de las gentes que navegaban desde Egipto. Pero ellos tuvieron su castigo cuando una flota se acercó y devastó su país» $(\mathrm{XVI}, 4,18)$.

Son las dos únicas referencias a las actividades marítimas de los nabateos, conocidos por su control de las rutas caravaneras, pero no precisamente por sus experiencias marineras. La cuestión es: ¿actuaron realmente los nabateos como piratas? A juzgar por los testimonios de las fuentes antiguas parece que sí. Pero, ¿cuándo tuvieron lugar estos sucesos de los que no se vuelve a hablar? Es imposible determinarlo con exactitud y se han propuesto diversas fechas, aunque parece evidente que sucedieron durante la época ptolemaica, dada la mención a «los reyes de Alejandría».

Sin embargo, no se conocen apenas referencias sobre las relaciones de nabateos y Ptolomeos durante el período helenístico.

La intervención nabatea en el comercio de los productos aromáticos está documentada ya desde fines del siglo IV a.C. pues Diodoro (siguiendo en esta ocasión a Jerónimo de Cardia), narra el ataque que Antígono Monoftalmos ordenó llevar a cabo contra los árabes nabateos y comenta que:

«Aunque hay muchas tribus árabes que usan el desierto como pasto, los nabateos los sobrepasan en riqueza aunque no son muchos más que diez mil de número; no pocos de ellos llevan al mar incienso y mirra y las más valiosas clases de especias, que obtienen de aquellos que las traen de Arabia Eudaemon» (XIX, 94, 4-5).» 
Además, estos nabateos explotaban también el asfalto del Mar Muerto:

«Hay también un gran lago que produce gran cantidad de asfalto, del cual obtienen no pocos ingresos» (II, 48,6-7)

Y esta otra riqueza atrajo igualmente a Antígono quien envió al propio Jerónimo de Cardia para que preparara barcos y recogiera el asfalto. Sin embargo, la empresa resultó fallida:

«Pues los árabes, reuniendo un número de 6.000 y navegando en sus balsas de caña contra aquellos de los barcos, mataron a casi todos con sus flechas» (XIX, 94,100).»

Esta es la única ocasión en la que se menciona a los nabateos construyendo balsas hechas con cañas, cosa lógica pues la madera no abunda precisamente en la región.

Los beneficios que los nabateos obtenían de su comercio debían ser importantes, a juzgar por el botín que las tropas de Antígono obtuvieron en su primer ataque:

"Cogió la mayor parte del incienso y la mirra y unos 500 talentos de plata» (XIX, 95).»

También Plutarco destaca que los griegos recogieron de los árabes nabateos un gran botín y 700 camellos, antes de dar término a su expedición (Demetrio, 7,1).

Pero volvamos a las relaciones entre los nabateos y los Ptolomeos. Si se acepta que la fuente de Diodoro, en su relato de las actividades de los nabateos como piratas, fue Agatárquides de Cnido y su obra perdida Sobre el Mar Eritreo, esto puede situarnos en la segunda mitad del siglo II a.C. Por aquel entonces la capital nabatea de Petra había adquirido ya una reputación internacional como emporio prominente. Esto se refleja, por ejemplo, en el viaje realizado en el año 120 a.C. por Moschio, hijo de Kydimos, un embajador de Priene, cuya misión diplomática en el este del Mediterráneo incluyó visitas a Alejandría y a «Petra en Arabia».

Los pocos autores modernos que se han ocupado de la cita de Diodoro sobre los piratas nabateos, relacionan estos sucesos con el comercio marítimo que se desarrolló como resultado del descubrimiento de los monzones estacionales, vientos que permitían a los barcos que negociaban con la India ir hasta allí sin efectuar escalas, es decir, sin necesidad de realizar un largo y costoso viaje por la costa.

Así, G. W. Bowersock ${ }^{1}$, sitúa el enfrentamiento entre nabateos y Ptolomeos en el siglo II a.C. y comenta: «La aparición de la piratería nabatea fue probablemente debida a la preocupación por el nuevo comercio marítimo que los egipcios estaban

1 En Roman Arabia, 1983, p. 21. 
desarrollando como resultado del descubrimiento de los monzones. Los barcos cargados con especias y perfumes podían acudir directamente a los puertos de Egipto en las estaciones apropiadas, y desde allí los productos podían dirigirse al Mediterráneo. Los nabateos debieron darse cuenta pronto de que el nuevo tráfico por mar significaba un declive gradual para el comercio terrestre, que era la base de su prosperidad». Y añade: «La piratería de los nabateos debe ser contemplada, por tanto, en el contexto del creciente comercio entre los centros de especias y perfumes del este y los puertos egipcios de la costa oeste del Mar Rojo».

J. F. Healey sitúa aún más tarde los acontecimientos que nos ocupan pues afirma: «El poderío comercial de los nabateos fue en última instancia vulnerable por la posibilidad del traspaso del comercio a las rutas del Mar Rojo-Egipto y el Golfo Árabe-Palmira en el mundo romano. Este incremento está explícitamente referido como una alternativa en Estrabón XVI, 4,24 y puede ponerse en conexión con el relato de algunos nabateos convirtiéndose en piratas. El desarrollo de otras rutas contribuyó al rápido declive de los nabateos tras el año 106 d.C.»²

En una obra más reciente, M.G. Amadasi y E. Equini ${ }^{3}$, siguen la hipótesis de Bowersock y conectan la piratería nabatea con el comercio marítimo que se desarrolló como resultado del descubrimiento de los monzones estacionales: «Gracias a los vientos seguros, las mercancías desde el este podían navegar por la costa del Mar Rojo, dominada por los nabateos, hasta alcanzar Egipto directamente por barco, y desde allí continuaban hacia el Mediterráneo sin tener que cruzar el interior de la ruta de Petra-Gaza. Esto significa que la piratería nabatea quizás derivara, al menos en parte, del deseo de proteger sus propias rutas comerciales».

Debemos plantearnos dos cuestiones. La primera es ¿cuándo se descubrieron los monzones que permitieron cambiar los viajes comerciales a los egipcios y que, por tanto, pudieron afectar negativamente a las empresas comerciales caravaneras nabateas? No se sabe con exactitud pues el primer documento que los menciona es el famoso Periplo del Mar Eritreo, una obra tardía para el tema que nos ocupa, que se sitúa en el siglo I d. C.: según Periplo 26, está claro que el comercio de tránsito había cesado y que se navegaba directamente a la India utilizando los monzones.

Por otro lado, es seguro que se usaban los monzones en época de la expedición del prefecto de Egipto, Elio Galo, al sur de Arabia ordenada por Augusto4, pues Estrabón dice que grandes barcos navegaban a la India desde comienzos de la ocupación romana en Egipto (II, 5, 12).

Y la segunda cuestión es si realmente el descubrimiento de los vientos de los monzones supuso un declive del comercio nabateo, que requirió una respuesta en forma de actividades piratas.

2 The Religion of the Nabataeans. A Conspectus, 2001, p. 33.

3 Petra, 2002, p. 24.

4 Esta audaz empresa, que acabó en un desastre, tuvo lugar en el 26 a.C. con la ayuda de unos 1.000 nabateos y estuvo guiada por Sileo, ministro del rey nabateo. Vid., Estrabón, IV, 22-24. 
En principio, parece que el comercio de productos aromáticos y de especias nabateo a través de Petra pudo haberse visto afectado por el crecimiento del comercio egipcio en el Mar Rojo. Estrabón $(\mathrm{XVI}, 4,24)$ dice que los productos aromáticos que anteriormente llegaban al puerto de Leuke Kome (y de allí a Petra) habían sido en su mayor parte redirigidos a través de Myos Hormos en su época:

«Entonces los cargamentos llegaban desde Leuke Kome a Petra y de allí a Rhinocolura en Fenicia cerca de Egipto, y desde allí a otros lugares. Pero ahora la mayoría son llevados a Alejandría por el Nilo, traídos hasta Myos Hormos desde Arabia e India.»

Según esta información de Estrabón, parece que los nabateos perdieron al menos parte del comercio, pues había barcos que usaban los puertos egipcios del Mar Rojo, y algunos investigadores consideraron que era causa suficiente para suponer la existencia de un declive del comercio nabateo en el siglo I d.C. Abraham Negev ${ }^{5}$ y otros añadieron a este testimonio literario algunas evidencias arqueológicas y numismáticas que parecían confirmar tal declive. Las causas para elegir esta otra ruta se atribuían tanto al coste menor del transporte marítimo como a la deliberada política económica de la administración romana.

Junto al testimonio de Estrabón, las evidencias arqueológicas consistían en prospecciones de superficie en la ruta entre Petra y los puertos del Mediterráneo (Petra-Gaza), así como en excavaciones realizadas en las ciudades nabateas del Negev, como Obodas y Mampsis. Parecía existir una falta de actividad en la ruta a mediados del siglo I d.C., que coincidía con destrucciones en las ciudades, presumiblemente a causa de tribus nómadas. Además, en las monedas nabateas se apreciaba una disminución del contenido de plata en el siglo I d.C.: un $20 \%$ en los reinados de Malco II (últimos años) y durante todo el reinado de Rabel II. Según Negev, todo esto se debería al declive en el comercio de las especias, combinado con disturbios nómadas, lo que habría conducido a la decadencia de la prosperidad nabatea. Negev también exponía que esta pérdida de ingresos fue parcialmente corregida por la colonización del Hauran y la creciente importancia de Bostra a expensas de Petra. Él situaba el cese del comercio en el año 7 d.C., basándose en la fuerte disminución en el contenido de plata de las monedas nabateas en esta fecha concreta.

Pero conviene matizar toda esta información. En primer lugar, Estrabón se está refiriendo solo al comercio por mar que iba por Leuke Kome, no a todo el comercio, que incluía también rutas terrestres. Aunque los barcos fueran a los puertos egipcios de Berenice y Myos Hormos en vez de a Leuke Kome, esto no afectó decisivamente a las rutas caravaneras. El testimonio de Estrabón de que había declinado el tráfico por Leuke Kome no puede considerarse una prueba del declive general de la fortuna de los nabateos, pues no comenta nada acerca del comercio terrestre de la «Ruta del incienso» o del que cruzaba Arabia desde la localidad de Gerrha.

5 NEGEV, A.: «The Date of the Petra-Gaza Road», PEQ 98 (1966), pp. 89-98. 
Existen considerables pruebas de que las rutas terrestres del comercio del incienso continuaron siendo de importancia primordial para los nabateos. Plinio (H.N. 6, 26; 12, 32) habla del comercio terrestre y del marítimo e indica que el comercio marítimo hacia Egipto existía al mismo tiempo que el terrestre hacia el reino nabateo.

Por otro lado, la ocupación nabatea de Hegra (actual Medain Saleh, Arabia Saudí) en el Hejaz, muestra que el comercio terrestre del incienso no había desaparecido en el siglo I d.C., sino que mantenía su vitalidad al menos hasta este período. Hegra fue un gran puesto militar nabateo a fines del siglo I a.C., y esto está atestiguado por las tumbas talladas en la roca de los oficiales militares, que pueden datarse por las inscripciones: la tumba más antigua data del año 1 d.C. y la última del 75 d.C. Aunque se han sugerido varias teorías para el propósito de este puesto, como posición de reserva en caso de invasión romana del reino ${ }^{6}$, o puesto defensivo de la frontera ${ }^{7}$, parece más probable que un lugar de tal tamaño tan lejos del centro del reino nabateo sirviera para proteger una valiosa instalación. Y esta no puede ser otra más que el comercio del incienso y la mirra.

Asimismo, el hecho de que la última inscripción pertenezca al año 75 d.C. no significa que Hegra fuera abandonada en esta época, sino sólo que cesaron las construcciones de tumbas lujosas. Los enterramientos posteriores pudieron hacerse en las tumbas familiares existentes, o quizás la guarnición se redujo o movió por alguna razón. La importancia de Hegra debida al comercio continuó y no perdió su importancia y prueba de ello es la ocupación romana del lugar tras la anexión en el 106 d.C., que se conoce por los hallazgos de inscripciones romanas en el lugar.

Finalmente, hay que destacar que la mirra y el incienso, los dos productos más lucrativos para el comercio de los nabateos, provenían del sur de Arabia y, por tanto, no se vieron afectados por el incremento de los puertos egipcios en el Mar Rojo, ni por las nuevas rutas de navegación a la India que aprovechaban los vientos de los monzones.

De manera que se puede afirmar que el tráfico terrestre de la «Ruta del incienso» desde el sur de Arabia continuó fluyendo hasta al menos los primeros tres cuartos del siglo I d.C. (tal y como atestigua la prosperidad del enclave nabateo de Hegra), y probablemente hasta mucho después.

Pero además, parece que el comercio continuó, al menos en cierta medida, por Leuke Kome, pues el Periplo 19 se refiere a los pequeños barcos árabes que usaban el puerto y a que había una guarnición y un cobrador de impuestos allí. Como la fecha del Periplo se sitúa a mediados del siglo I d.C., y es posterior por tanto a Estrabón, esta referencia muestra que Leuke Kome no estaba totalmente fuera de uso. Al contrario, se usaba lo suficiente como para requerir unas tropas apostadas allí y un recaudador de impuestos. Aunque su importancia hubiera disminuido

6 BOWERSOCK, G.: Roman Arabia, Harvard, 1983, p. 57.

7 BOWSHER, J.: «The Frontier Post of Medain Saleh», p. 27, en P. Freeman y D. Kennedy (eds.), The Defence of the Roman and Byzantine East I, Oxford (1986), pp. 23-29. 
desde el siglo I a.C. Leuke Kome no era ciertamente una ciudad fantasma en la época en que se escribió el Periplo.

En cuanto al aparente declive de los lugares y rutas en la región del Negev, que se ha aducido también como prueba del declive de Petra en el siglo I d.C., es incorrecto $^{8}$. Las excavaciones han mostrado que las vías comerciales continuaban en uso durante el siglo I d.C. y así siguieron en el período romano tras la anexión. También las excavaciones en la ciudad de Obodas han mostrado que seguía ocupada. Tanto las autoridades nabateas como las romanas protegían esta ruta que unía Petra con Gaza, pues hubo presencia militar continuada en varios sitios. Por tanto, no hay por qué pensar en una decadencia de Petra debido a la falta del comercio en el siglo I d.C. Si hubo incursiones nómadas fueron efímeras y limitadas. No hubo interrupción del comercio del incienso, o al menos no hay tangibles evidencias de ello.

Parece lógico pensar, por tanto, que la demanda de especias y otros productos exóticos era tan grande que los nuevos puertos construidos o agrandados en el Mar Rojo egipcio a comienzos del período romano simplemente complementaron el floreciente comercio transarábigo terrestre conducido por los nabateos.

Por otro lado, N. Groom ${ }^{9}$ ha demostrado que esto queda confirmado por el ritmo de la cosecha del incienso con relación al tiempo de navegación óptimo del Mar Rojo, combinado con el gran incremento de la demanda de aromáticas que causó el comienzo de una segunda cosecha. El tiempo de cosecha del incienso lo cuenta Plinio (N.H. 12, 32):

"Cuando había menos oportunidades de vender, era la costumbre de reunirla; pero ahora el beneficio lo trae otra cosecha. La anterior y natural cosecha se producía alrededor de la salida de la constelación del perro.»

«El cultivo veraniego es recolectado en el otoño: es el más puro y es blanco. La segunda cosecha es en primavera, la corteza se corta en el invierno para este propósito; la savia roja viene de ésta, no se puede comparar con la primera.»

Cuando se comparan los tiempos de cosecha de Plinio con los tiempos de navegación mencionados en el Periplo, surge un diseño de comercio que puede explicar la supervivencia del comercio terrestre, incluso aunque el comercio del Mar Rojo fuera predominante. El Periplo $(34,49,56)$, deja bien claro que los barcos que navegaban hacia la India no podían participar en la mejor cosecha de incienso, la del otoño, pues viajaban desde Egipto en julio y no volvían hasta la época de la segunda cosecha de primavera. De forma que podrían tomar algo de esta cosecha inferior en Moscha Limen en su viaje de vuelta. La etapa para colectar la cosecha de otoño de mirra e incienso debía organizarse separadamente, comenzando en septiembre y volviendo con los monzones del noreste, que usualmente comienzan a mediados de octubre.

8 COHEN, R.: «New Light on the Petra-Gaza Road», Bibl. Arch. 45,3 (1982), pp. 240-47.

9 Frankincense and Myrrh. A Study of the Arabian Incense Trade, 1981. 
En cambio, las caravanas del incienso, que no tenían que esperar hasta el comienzo de los monzones, podían llegar a Gaza por la misma época en que los barcos estaban llegando al sur de Arabia. Por tanto, la habilidad de los comerciantes terrestres para llevar su incienso al mercado antes que los comerciantes marítimos puede ser una buena razón para la supervivencia del comercio terrestre, aunque la alternativa marítima fuera más barata.

La teoría, ampliamente defendida, de que el tráfico marítimo reemplazó el terrestre, que resultaba más caro, a comienzos del siglo I d.C. es, por tanto, errónea.

Una vez confirmado que el comercio de especias continuó desarrollándose por vía marítima a través de Leuke Kome y, sobre todo, terrestre, a pesar del descubrimiento de los monzones y del incremento del comercio por los puertos egipcios del Mar Rojo, parece que no tiene mucho sentido que esta fuera la causa de que los nabateos atacaran los barcos egipcios.

Además, ¿acaso los monzones eran conocidos ya en época ptolemaica, cuando tuvieron lugar los enfrentamientos entre nabateos y los «reyes de Alejandría»? A pesar de que resulta imposible con los datos de que disponemos actualmente fechar el descubrimiento de los monzones ${ }^{10}$, seguramente la respuesta es negativa. De manera que volvemos a la época helenística y a los gobernantes ptolomeos.

Antes de la llegada de los romanos, los Ptolomeos habían comenzado ya a explotar el comercio con Arabia y la India construyendo puertos a lo largo de la costa del Mar Rojo, aunque su magnitud no sería tan grande como el que se produciría después, bajo el poder de Roma, tal y como indica Estrabón (XVII, 1,13).

Al principio, el comercio ptolemaico llegaba solo hasta Eudaemon (Aden) y los puertos cerca de los estrechos de Bab el-Mandeb, donde los barcos egipcios podían intercambiar mercancías con aquellos que venían desde la India y el Golfo Pérsico y así sucedió hasta el descubrimiento de los monzones ${ }^{11}$.

Ptolomeo II Filadelfos fue el primer soberano egipcio en estimular al comercio regular con el norte de Arabia y el Africa subsahariana, y promovió la construcción de puertos en las costas egipcias del Mar Rojo (como el de Berenice), en el Sudán y en Etiopía, un empeño que sería continuado por sus sucesores: Ptolomeo III Evergetes y Ptolomeo IV Filopator. En esta época, a lo largo del siglo III a.C., los nabateos ya estaban involucrados en el comercio terrestre de las especias que llevaban «hasta el mar», según el testimonio de Diodoro ${ }^{12}$ y también en el del asfalto del Mar Muerto, que transportaban hasta Egipto, donde se vendía para embalsamar los cadáveres ${ }^{13}$. Todo esto implica una organización sólida, que algunos

10 Vid., RASCHKE, M. G.: «New Studies in Roman Commerce with the East», ANRWII, 9,2 (1978), pp. 660-663. p. 27.

11 MURRAY, G.W. y WARMINGTON, E. H.: «Trogodytica: The Red Sea Litoral in Ptolemaic Times»,

12 Diodoro, XIX, 94, 4-5.

13 Diodoro, XIX, 98. 
autores actuales consideran impropia de los nabateos en este período temprano de su historia. Sin embargo, cada vez se conocen más datos referentes a los siglos III y II a.C., que están cambiando, poco a poco, la imagen que se tenía sobre los nabateos.

Un buen ejemplo de ello son los epigramas de Posidipo de Pella -fechados a mediados del siglo III a.C.- donde se menciona a un nabateo rey de los árabes quien combate a caballo ${ }^{14}$, lo que parece implicar la existencia de una organización estatal ya en esa época.

Sin embargo, con los datos de que disponemos resulta imposible establecer si los testimonios de Diodoro (III, 43, 4-5) y Estrabón (XVI, 4,18) sobre la participación de los nabateos en prácticas de piratería en el Mar Rojo, que uno o varios de los reyes Ptolomeos del vecino Egipto suprimieron drásticamente, fueron genuinas actividades de piratería o enfrentamientos debidos a la competición comercial marítima.

\section{BIBLIOGRAFÍA}

AMADASI, M.G. y EQUINI, E.: Petra, Chicago, 2002.

BASTIANINI, G. y GALLAZZI, C.: Posidippo di Pella Epigrammi P. Mil. VogI. VII, 309, Milán, 2001.

BOWERSOCK, G.: Roman Arabia, Harvard, 1983.

BOWSHER, J.: «The Frontier Post of Medain Saleh» en P. Freeman y D. Kennedy (eds.), The Defence of the Roman and Byzantine East I, Oxford (1986), pp. 23-29.

COHEN, R.: «New Light on the Petra-Gaza Road», Bibl. Arch. 45,3 (1982), pp. 240-47.

GRAF, D. y SIDEBOTHAM, S.: «Nabataean Trade» en G. Markoe (ed.), Petra Rediscovered, Cincinnati (2003), pp. 65-74.

GROOM, N.: Frankincense and Myrrh. A Study of the Arabian Incense Trade, Londres, 1981.

MURRAY, G.W. y WARMINGTON, E.H.: «Trogodytica: The Red Sea Litoral in Ptolemaic Times", The Geographical Journal, 133, no1 (1967), pp. 24-33.

NEGEV, A.: «The Date of the Petra-Gaza Road», PEQ 98 (1966), pp. 89-98.

RASCHKE, M.G.: «New Studies in Roman Commerce with the East», ANRW II, 9,2 (1978), pp. 604-1378.

YOUNG, G.K.: Rome s Eastern Trade, Londres, 2001.

${ }^{14}$ BAStIANINI, G. y GALLAZZI, C.: Posidippo di Pella Epigrammi P. Mil. Vogl. VII, 309, Milán, 2001. 
